\title{
Modeling and Performance Analysis for Cell Access and Handoff Schemes in Two-Tier Cellular Networks
}

\author{
Kyungkoo Jun ${ }^{1}$ and Sijung Kim ${ }^{2}$ \\ ${ }^{1}$ Department of Embedded Systems Engineering, Incheon National University, Incheon 406-772, Republic of Korea \\ ${ }^{2}$ Department of Entertainment Engineering and Design, University of Nevada, Las Vegas, NV 89154, USA
}

Correspondence should be addressed to Kyungkoo Jun; kjun@incheon.ac.kr

Received 7 September 2014; Accepted 3 November 2014; Published 24 November 2014

Academic Editor: Marco Anisetti

Copyright ( $\odot 2014$ K. Jun and S. Kim. This is an open access article distributed under the Creative Commons Attribution License, which permits unrestricted use, distribution, and reproduction in any medium, provided the original work is properly cited.

\begin{abstract}
We investigate the effects of handoff on system performance in two-tier cellular networks. Two of the main performance metrics are new call blocking probability and handoff drop rate. We develop analytical models to evaluate the performance of two different handoff schemes. One scheme considers only femto-to-macrocell handoff while the other is bidirectional including macro-tofemtocell handoff. Our model is more elaborate than existing ones which have not considered the mobility of mobile stations. Numerical results show that the bidirectional scheme performs better than the femto-to-macrocell handoff as it achieves lower blocking probability and drop rate.
\end{abstract}

\section{Introduction}

Two-tier wireless networks [1-3] have been proposed to accommodate the ever-increasing data traffic in various outdoor and indoor environments by supporting high throughput and improving the energy efficiency of wireless communications [4-6]. The two-tier networks are configured by including femtocells within the coverage of a macrocell. To this end, network operators need to choose cell access scheme. They can have mobile stations try femtocells first or macrocells when accessing network resources. We believe it is worth developing mathematical model of different access schemes to evaluate performance and understand further the characteristics of the two-tier networks.

Various research efforts regarding the performance of the two-tier network have appeared in [7-9]. Spectrum-sharing schemes between macrocell and femtocell, as well as among femtocells, have been proposed to improve spatial reuse gain in [7]. The schemes limit the maximum intensity of simultaneously transmitting femtocells that satisfy a given per-tier outage constraint. Stochastic geometry is used to model and analyze performance of two-tier networks in a multichannel environment [8]. The proposed model quantifies the performance gain in outage probability and shows the existence of an optimal spectrum sensing threshold. A semidistributed interference management scheme is proposed in [9]. The management of both the cross-tier and cotier interferences is a critical performance obstacle for two-tier cellular networks.

Different studies on the cell access scheme in twotier networks have appeared in the literature $[1,10,11]$. An incentive mechanism to motive the femtocell operators to open portion of the access opportunities to macrousers was proposed in [1]. The users in two-tier networks choose service cells dynamically according to the performance satisfaction level, pricing, and spectrum sharing schemes between macrocells and femtocells. Comprehensive investigation is given on the key aspects and challenges in two-tier networks [10]. Particularly, cell identification schemes, access control schemes, cell search, and handover schemes were discussed and three representative algorithms were described. Poisson point process was proposed to model the coverage of macrocells and femtocells in [11]. It proposes an approximation to the coverage probability for the open access and closed access policies, respectively.

An analytical model for two-tier networks has been proposed in [12]. Its model analyzes the performance of different cell access schemes from the traffic perspective. However, it has not considered in its analysis the movement of the mobile 
stations; thus, the handoff is not taken into account. A model with soft handoff was proposed in CDMA cellular networks [13]. The application of the model to the two-tier networks is difficult due to the different characteristics of femtocells overlaying macrocells.

In this paper, we develop a comparative performance evaluation study of two different types of the handoffs that are particular in two-tier wireless networks. One is the handoff from femtocells to a macrocell, called femto-to-macrocell handoff. The other is the opposite directional handoff, that is, from a macrocell to femtocells, macro-to-femtocell handoff. They are different from each other when handoff attempts fail. The femto-to-macrocell handoff drops the failed calls while the macro-to-femtocell handoff has the calls to continue to be served through the macrocell. The femto-to-macrocell handoff should obviously drop the call when a mobile station moves beyond the coverage of femtocells, but a macrocell is not able to provide available channels. On the contrary, the macro-to-femtocell handoff does not necessarily drop the call even though a target femtocell does not have available channels because the call is still able to occupy the macrocell channel.

Considering the two different types of the handoffs, we propose two handoff schemes: femto-to-macrocell handoff and bidirectional handoff scheme. The former supports only the femto-to-macrocell handoff. The latter supports both directions of handoff. This work is different from [12] in that we consider handoffs in performance analysis.

We note that, for new call arrivals, both of the proposed schemes work in the same way as the femtocell priority scheme of [12]. In the scheme, if a new call arrives at the system, the femtocells try to admit the call if the mobile station is within the coverage of the femtocells and there are available channels before the macrocell attempts. Thus, the two proposed schemes are similar in that they deal with new call admission by giving preference to the femtocells.

The rest of the paper is organized as follows. Section 2 describes the system model of two-tier cellular networks. In Sections 3 and 4, two continuous time Markov chain models are proposed, which takes into account handoff activities. In Section 5, we define performance metric, the blocking probability, and the handoff drop rate and present the analysis results of the different handoff schemes. Section 6 concludes the paper.

\section{System Model}

Figure 1 shows a scenario of a two-tier wireless network. It consists of a single macrocell and several femtocells. There are three different types of functional units involved: the macrocell base station (mBS), femtocell base stations (fBSs), and mobile stations (MSs). We assume that the mBS provides $C_{m}$ channels to the MSs and its coverage area is $S_{m}$, while there are $N$ femtocells randomly distributed within the macrocell coverage area. The coverage of femtocells does not overlap with each other. We denote by $S_{i}$ the coverage area of the fBS $i$ and by $C_{i}$ the number of channels: $1 \leq$ $i \leq N$. As for the workload, it is assumed that new call

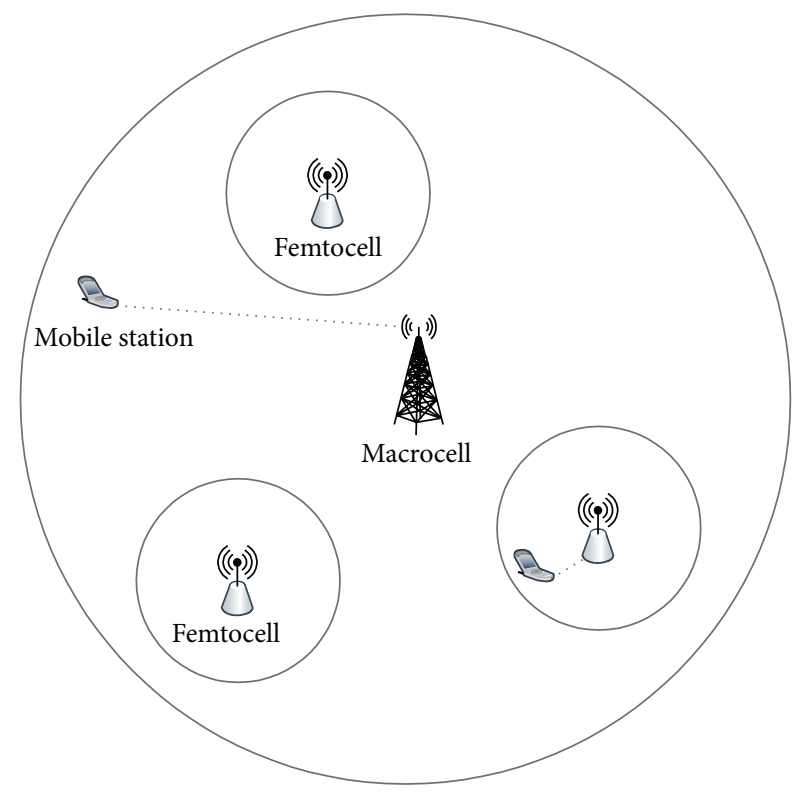

FIgURE 1: Two-tier cellular network consisting of a macrocell and femtocells.

admissions are attempted following Poisson process of rate $\lambda$ and call durations are exponentially distributed with rate $\mu$. An ongoing call is supposed to occupy one channel at any moment. Since our model considers mobility of MSs, the handoff interval of accepted calls follows an exponential distribution with mean $\beta$.

However, for simplicity of our analysis, we assume that the MSs do not move beyond the coverage area of the mBS. Finally, an MS is able to access both $\mathrm{mBS}$ and any $\mathrm{fBS}$ as long as they are within its communication range.

\section{Modeling of Handoff-Considered Schemes}

We model the two proposed schemes as a birth-and-death process [14]. For brevity of explanation, we consider a system with a single macrocell and a single femtocell. We note that larger systems can be modeled based on this simple version without difficulties. As in [12], let vector $\left(x_{m}, x_{i}\right)$ represent one of the states of the system. We denote by $x_{m}, 0 \leq x_{m} \leq$ $C_{m}$, the number of ongoing calls at the mBS and by $x_{i}, 0 \leq$ $x_{i} \leq C_{i}$, the number of ongoing calls at the fBS $i$. We note that the set of the state vectors and the transition relationship among them define an irreducible and ergodic continuous time Markov chain.

Before describing our model that considers handoff, we present the model without handoff. Figure 2 shows the state transition diagram of the femtocell priority scheme [12]. For new call arrivals, both of our proposed schemes behave in the same way as in Figure 2. The transitions are as follows:

(1) $\left(x_{m}, x_{i}\right) \rightarrow\left(x_{m}, x_{i}+1\right)$ : a new call arrives within the coverage area of the fBS $i$ and it is accepted by the fBS $i$; 


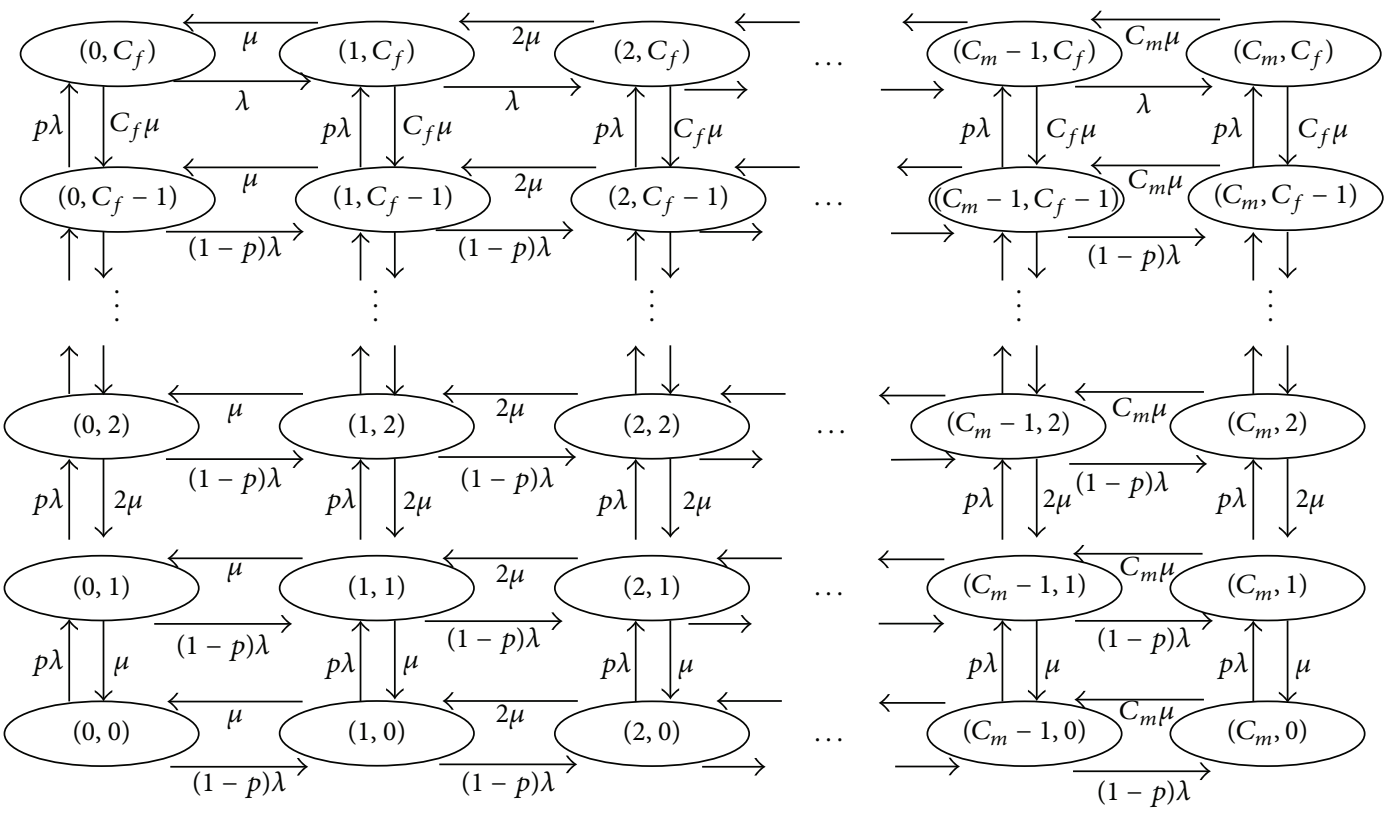

FIGURE 2: State transition diagram of cell access scheme which tries femtocells first.

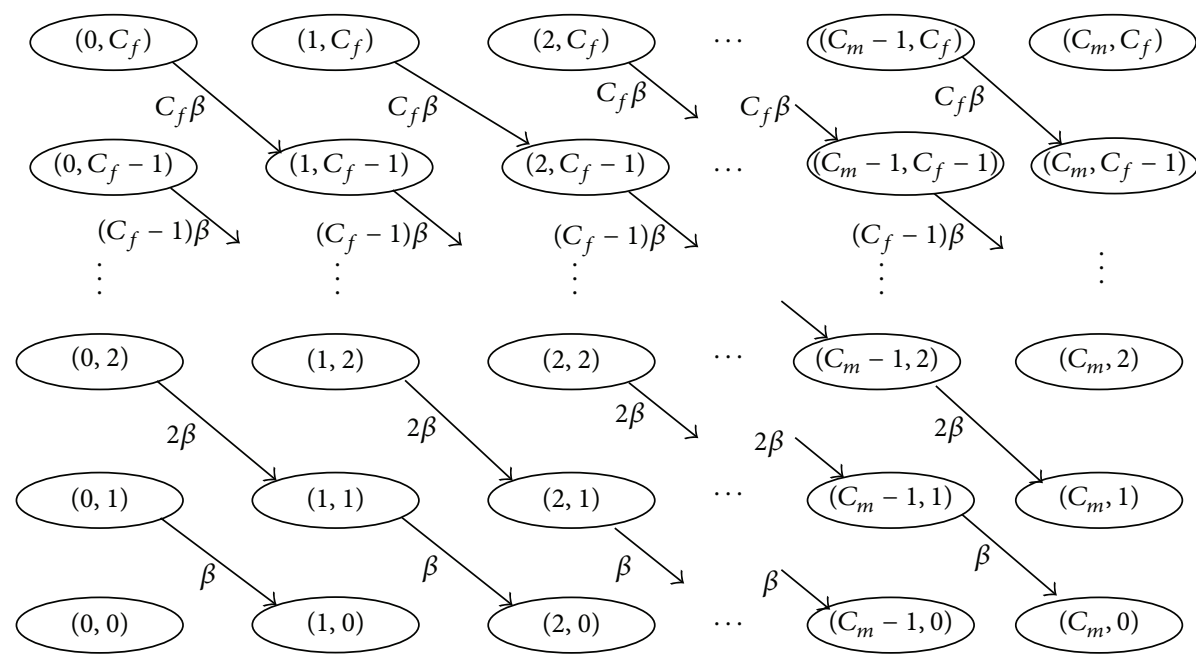

FiguRE 3: State transition diagram of femto-to-macrocell handoff scheme.

(2) $\left(x_{m}, C_{i}\right) \rightarrow\left(x_{m}+1, C_{i}\right)$ : a new call arrives within the coverage area of the fBS $i$ but it is accepted by the mBS because all the channels of the fBS $i$ are occupied;

(3) $\left(x_{m}, x_{i}\right) \rightarrow\left(x_{m}+1, x_{i}\right)$ : a new call arrives outside the coverage area of the fBS $i$ and it is accepted by the $\mathrm{mBS}$;

(4) $\left(x_{m}, x_{i}\right) \rightarrow\left(x_{m}, x_{i}-1\right)$ : a call of the fBS $i$ terminates;

(5) $\left(x_{m}, x_{i}\right) \rightarrow\left(x_{m}-1, x_{i}\right):$ a mBS call terminates.

Besides the set of the transitions of Figure 2, we add to the proposed model the transitions related to the femto-tomacrocell handoff. Figure 3 shows the transition diagram of the handoff. A mobile station which has an ongoing call moves out of the femtocell and makes handoff to a macrocell. The transitions are as follows:

$$
\left(x_{m}, x_{i}\right) \longrightarrow\left(x_{m}+1, x_{i}-1\right),
$$

where $0 \leq x_{m} \leq C_{m}-1$ and $1 \leq x_{i} \leq C_{f}$. A mobile station makes a handoff to the macrocell from the fBS $i$.

The macro-to-femtocell handoff adds other transitions to the model as shown in Figure 4. A mobile station which has an ongoing call in the macrocell moves to a femtocell and makes handoff. The transitions are as follows:

$$
\left(x_{m}, x_{i}\right) \longrightarrow\left(x_{m}-1, x_{i}+1\right) \text {, }
$$

where $1 \leq x_{m} \leq C_{m}$ and $0 \leq x_{i} \leq C_{f}-1$. A mobile station makes a handoff to the fBS $i$ from the macrocell. 


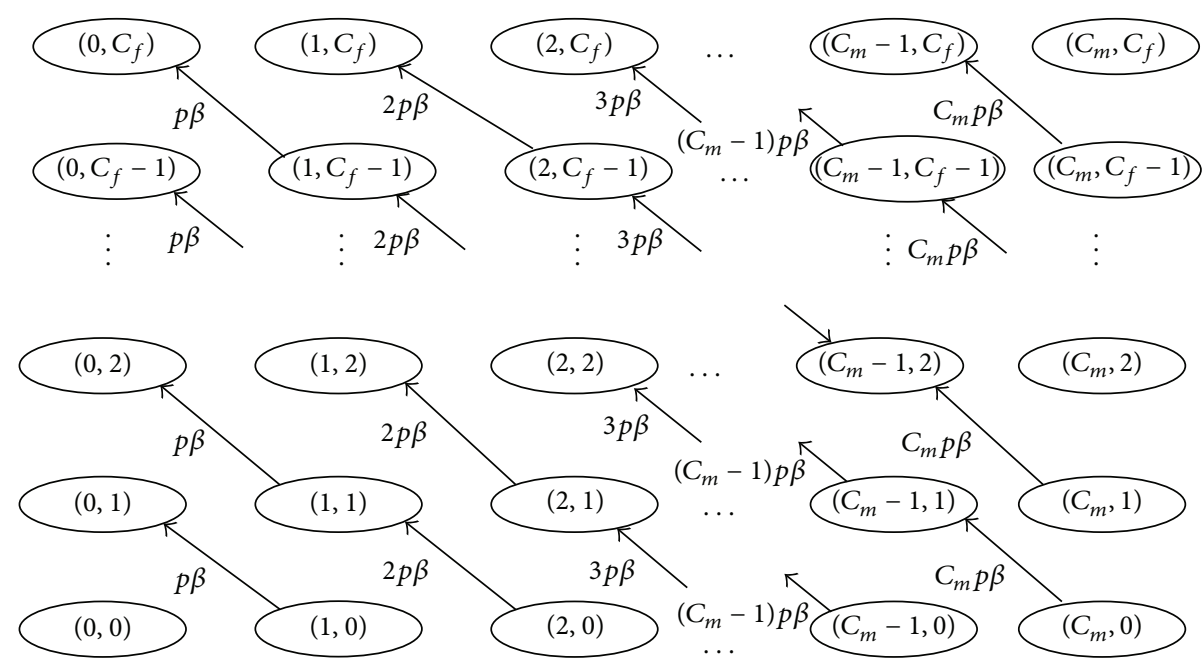

FIGURE 4: State transition diagram of macro-to-femtocell handoff scheme.

From the above transition diagrams, we now model the transition diagram of the femto-to-macrocell scheme by overlaying the transition diagram of Figure 3 on top of the diagram of Figure 2. In a similar way, the transition diagram of the bidirectional scheme is obtained by overlaying all the three transition diagrams.

In the rest of the paper, we identify the states not only by $\left(x_{m}, x_{f}\right)$ but also by assigning sequential numbers to them starting from 1 . We denote by $S$ the set of all the states of the system $S=\{1,2, \ldots, N\}$ where $N$ is the number of the states.

We need $\Pi$, the stationary state distribution of all the states, to analyze the performance. Let $\pi(i)=\pi\left(x_{m}, x_{f}\right)$ be the steady state probability of finding the system in state $i=\left(x_{m}, x_{f}\right)$. Let $P\left\{x_{t}=i\right\}$ be the probability of finding the system in state $i$ at time $t$. Then, we can define $q_{i j}$ as the probability that the system makes a transition from state $i$ to $j$ as follows:

$$
q_{i j}=\lim _{\Delta t \rightarrow 0} \frac{P\left\{x_{t+\Delta t}=j \mid x_{t}=i\right\}}{\Delta t}, \quad i \neq j
$$

which implies the transition rate or the intensity. We denote by $Q$ an $N \times N$ matrix that has $q_{i j}, 1 \leq i, j \leq N$, as its ( $i$ th, $j$ th) element. $Q$ is known to have the following properties [14]:

(1) $q_{i i} \leq 0$ for all $i \in S$;

(2) $q_{i j} \geq 0$ for all $i, j \in S$;

(3) $\sum_{j \in S} q_{i j}=0$ for all $i \in S$.

From the above, it is obvious that the total transition rate out of state $i, q_{i}$, is

$$
q_{i}=\sum_{j \neq i} q_{i j}
$$

and, for the equilibrium purpose, $q_{i i}$ is defined as $q_{i i}=-q_{i}$.
We can obtain $\Pi$ by solving the following global balance condition equations:

$$
\begin{aligned}
& \Pi \times Q=0, \\
& \Pi \times e^{t}=1,
\end{aligned}
$$

where $e=(1,1, \ldots, 1) \in R^{N}$.

Let $E$ be $N \times N$ matrix that every element is 1 . Then,

$$
\Pi \times E=e .
$$

We add (5) and (7) to obtain

$$
\Pi \times(Q+E)=e .
$$

From (8), $\Pi$ can be obtained by multiplying the inverse of $(Q+E)$

$$
\Pi=e \times(Q+E)^{-1}
$$

\section{Performance Metrics}

We consider two performance metrics to evaluate the proposed schemes. One is a new call blocking probability and the other is a handoff drop rate. To understand how these metrics are analyzed, we consider a system with one mBS and $N$ fBS. For simplicity, we assume that all the fBSs have the same number of channels; that is, $C_{i}=C_{f} \forall i$. Also, all of them have the same areas of the coverage; that is, $S_{i}=S_{f} \forall i$. We denote by $p$ the probability of finding an MS in the coverage area of $\mathrm{fBS} i, p=S_{f} / S_{m}$. We assume that the total coverage areas of the fBSs do not cover the whole macrocell by putting a constraint $N p<1$.

Recall that $\pi\left(x_{m}, x_{1}, x_{2}, \ldots, x_{N}\right)$ is the steady state probability of finding $x_{m}$ ongoing calls in the $\mathrm{mBS}$ and $x_{i}$ ongoing 
calls in the fBS: $1 \leq i \leq N$. Then, a new call blocking probability is given by

$$
\begin{aligned}
\mathrm{NBP}= & (1-N p) \pi\left(C_{m}, x_{1}, x_{2}, \ldots, x_{N}\right) \\
& +p \pi\left(C_{m}, C_{1}, x_{2}, \ldots, x_{N}\right) \\
& +p \pi\left(C_{m}, x_{1}, C_{2}, \ldots, x_{N}\right) \\
& +\cdots+p \pi\left(C_{m}, x_{1}, x_{2}, \ldots, C_{N}\right) .
\end{aligned}
$$

A new call is blocked when the corresponding MS is not covered by any of the $N$ fBSs and the mBS have all channels occupied or when it is within the coverage of $\mathrm{fBS}$, but all the channels of the $\mathrm{fBS}$ as well as the mBS are occupied. If we assume the uniform distribution of the fBSs within the $\mathrm{mBS}$ and the geographical occurrences of new call attempts, without loss of generality, the following holds:

$$
\pi\left(C_{m}, \ldots, C_{i}, \ldots\right)=\pi\left(C_{m}, \ldots, C_{j}, \ldots\right) \text { for } 1 \leq i, j \leq N .
$$

Thus, (9) is further simplified as

$$
\begin{aligned}
\mathrm{NBP} \approx & (1-N p) \cdot \pi\left(C_{m}, x_{1}, x_{2}, \ldots, x_{N}\right) \\
& +N \cdot p \cdot \pi\left(C_{m}, C_{1}, x_{2}, \ldots, x_{N}\right) .
\end{aligned}
$$

The other performance metric, the handoff drop rate, is given by

$$
\begin{aligned}
\mathrm{HDR}= & x_{1} \beta \pi\left(C_{m}, x_{1}, x_{2}, \ldots, x_{N}\right) \\
& +x_{2} \beta \pi\left(C_{m}, x_{1}, x_{2}, \ldots, x_{N}\right) \\
& +\cdots+x_{N} \beta \pi\left(C_{m}, x_{1}, x_{2}, \ldots, x_{N}\right) \\
= & \beta \sum_{i=1}^{C_{f}} i \pi\left(C_{m}, i, x_{2}, \ldots, x_{N}\right) \\
& +\beta \sum_{i=1}^{C_{f}} i \pi\left(C_{m}, x_{1}, i, \ldots, x_{N}\right) \\
& +\cdots+\beta \sum_{i=1}^{C_{f}} i \pi\left(C_{m}, x_{1}, x_{2}, \ldots, i\right) \\
= & \beta \sum_{j=1}^{N} \sum_{i=1}^{C_{f}} i \pi\left(C_{m}, i\right)_{j},
\end{aligned}
$$

where $\pi\left(C_{m}, i\right)_{j}$ is the sum of the steady state probabilities of finding $C_{m}$ macrocell calls and $i$ femtocell calls at $j$ th fBS.

A handoff call is dropped when an MS associated with a fBS moves beyond the coverage of the fBS, but the mBS has all channels occupied. Note that this metric is measured as rate not probability. We assume that the handoff occurs with rate $\beta$. Thus, the handoff dropping is inevitable when all $\mathrm{mBS}$ channels are occupied. It is more appropriate to measure how often the handoff occurs rather than whether it happens.

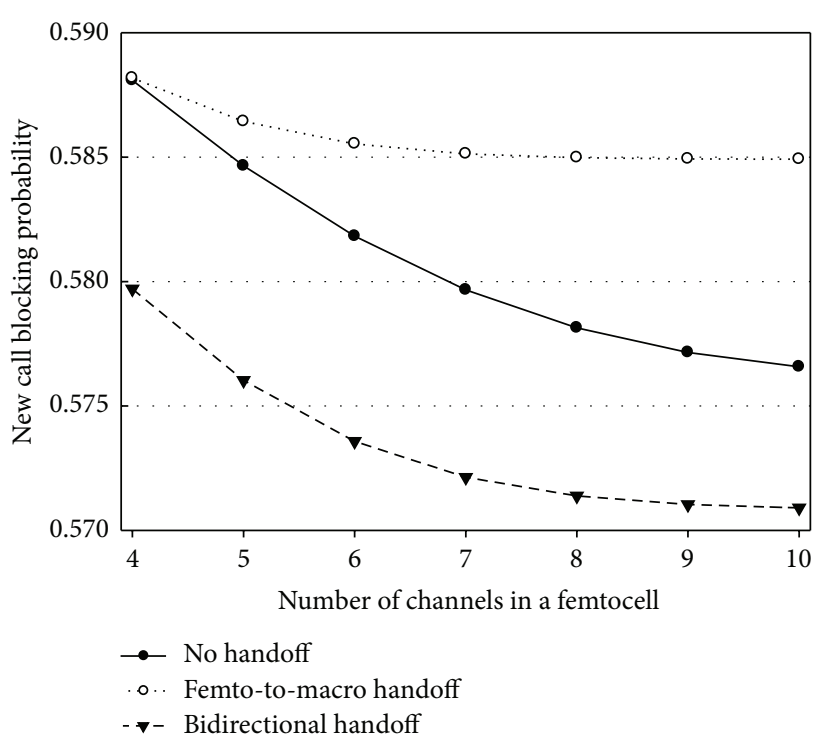

FIGURE 5: New call blocking probability in a system with four femtocells.

\section{Numerical Evaluation}

In this section, we compare the performance of the two handoff schemes studied in two-tier wireless networks. We also consider the scheme [3] which had not taken into account handoff. For evaluation, we define a network with the following parameters: $C_{m}=40, C_{i} \geq 4, \lambda=5, \mu=0.05, \beta=0.05$, $p=S_{f} / S_{m} \geq 0.05$, and $1 \leq N \leq 4$.

Figure 5 shows the changes of the new call block probability with the number of channels of the $\mathrm{fBS}$ in a simple scenario with one $\mathrm{mBS}$ and a single fBS. We denote by "no handoff" the femtocell priority scheme of [3] and by "femtoto-macro handoff" and "bidirectional handoff" the two proposed schemes, respectively. As expected, the blocking probabilities of all the three schemes decrease as the number of channels of the fBS increases. An interesting observation is that the blocking probability of no-handoff is analyzed lower than that of the femto-to-macro handoff scheme. It implies that handoff affects new call admission. The bidirectional handoff shows the lowest blocking probability proving that it is effective to utilize the resources of the system.

Figure 6 shows the evolution of the handoff drop rate with the growing coverage of a femtocell in the same scenario as above except that the number of the channels of the femtocell is 8 . We observe that the drop rate increases as the coverage area of the femtocell grows. Larger femtocell implies that more calls can be admitted to the femtocell. It increases the chances that the calls try handoff, resulting in higher handoff drop rate. It is interesting to observe that the drop rate of the bidirectional handoff scheme becomes lower than that of the femto-to-macro handoff scheme as the coverage area grows. It is explained by the fact that the larger area covering femtocell can accept more handoff calls from the macrocell, leaving rooms for the macrocell to accept the femto-to-macro handoff requests. 


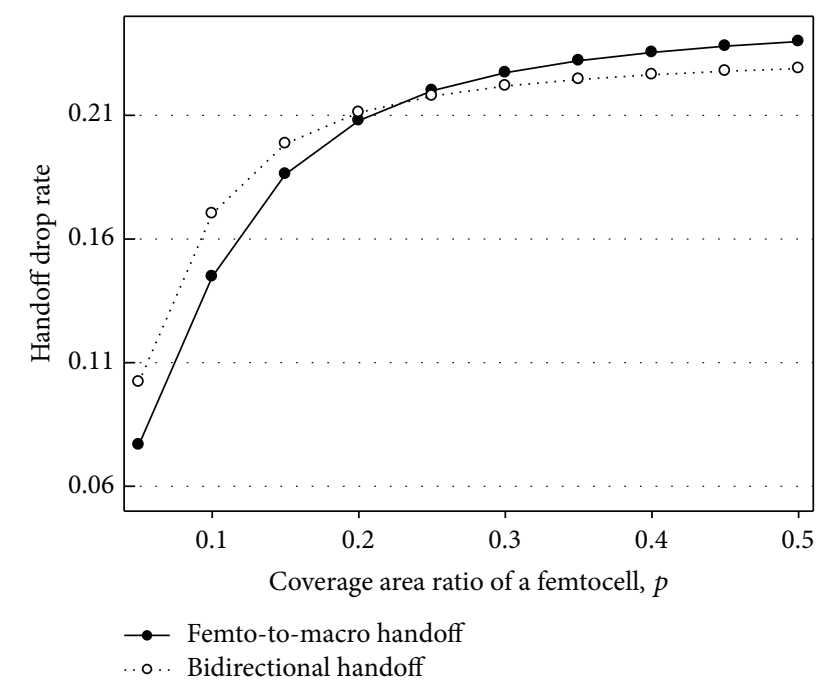

Figure 6: Handoff drop rate in a system with four femtocells.

\section{Conclusions}

We have modeled the two handoff schemes in a two-tier wireless network. One is the femto-to-macro handoff scheme where only handoff from femtocells to macrocells is permitted. Handoff drop occurs when a macrocell does not have available channel. The other scheme is the bidirectional scheme where the opposite direction handoff, that is, macrocell to femtocell, as well as femtocell to macrocell, is allowed. A similar work has modeled two cell access schemes. However, its model has not considered handoff for simplicity of the performance analysis. In this paper, we have modified the model to consider handoff.

We have modeled both schemes by continuous time Markov chain and defined two performance metrics: the new call blocking probability and the handoff drop rate. The numerical results showed that the modified model produced different results from the model without handoff, particularly in terms of the new call blocking probabilities. Comparing the two proposed schemes, as expected, the bidirectional scheme showed improved blocking probability than the femto-tomacro scheme.

The handoff drop rate is interesting in the sense that it is affected by the femtocell area. The drop rate of both schemes increases as the coverage area grows. The bidirectional scheme outperforms the femto-to-macro scheme as the femtocell coverage expands. Our results show that the model produces different outcomes depending on whether handoff is considered or not. Also, we note that the bidirectional scheme is superior to the femto-to-macro scheme because it is able to utilize the resources of femtocells more efficiently.

\section{Conflict of Interests}

The authors declare that there is no conflict of interests regarding the publication of this paper.

\section{Acknowledgment}

This work was supported by the University of Incheon International Cooperative Research grant in 2012.

\section{References}

[1] K. Zhu, E. Hossain, and D. Niyato, "Pricing, spectrum sharing, and service selection in two-tier small cell networks: a hierarchical dynamic game approach," IEEE Transactions on Mobile Computing, vol. 13, no. 8, pp. 1843-1856, 2014.

[2] Z. Zheng, X. Zhang, L. Cai, R. Zhang, and X. Shen, "Sustainable communication and networking in two-tier green cellular networks," IEEE Wireless Communications, vol. 21, no. 4, pp. 47-53, 2014.

[3] Y. S. Soh, T. Q. S. Quek, M. Kountouris, and G. Caire, “Cognitive hybrid division duplex for two-tier femtocell networks," IEEE Transactions on Wireless Communications, vol. 12, no. 10, pp. 4852-4865, 2013.

[4] Y. Jeong, H. Shin, and M. Z. Win, "Superanalysis of optimum combining with application to femtocell networks," IEEE Journal on Selected Areas in Communications, vol. 30, no. 3, pp. 509524, 2012.

[5] F. Wang and W. Wang, "Analytical evaluation of femtocell deployment in cellular networks using fractional frequency reuse," IET Communications, vol. 8, no. 9, pp. 1599-1608, 2014.

[6] J. Misic and V. B. Misic, "Bridge performance in a multitier wireless network for healthcare monitoring," IEEE Wireless Communications, vol. 17, no. 1, pp. 90-95, 2010.

[7] S.-M. Cheng, W. C. Ao, F.-M. Tseng, and K.-C. Chen, "Design and analysis of downlink spectrum sharing in two-tier cognitive femto networks," IEEE Transactions on Vehicular Technology, vol. 61, no. 5, pp. 2194-2207, 2012.

[8] H. Elsawy and E. Hossain, "Two-tier hetnets with cognitive femtocells: downlink performance modeling and analysis in a multichannel environment," IEEE Transactions on Mobile Computing, vol. 13, no. 3, pp. 649-663, 2014.

[9] A. Abdelnasser, E. Hossain, and D. I. Kim, "Clustering and resource allocation for dense femtocells in a two-tier cellular OFDMA network," IEEE Transactions on Wireless Communications, vol. 13, no. 3, pp. 1628-1641, 2014.

[10] D. Xenakis, N. Passas, L. Merakos, and C. Verikoukis, "Mobility management for femtocells in LTE-advanced: key aspects and survey of handover decision algorithms," IEEE Communications Surveys \& Tutorials, vol. 16, no. 1, pp. 64-91, 2014.

[11] C.-H. Lee and C.-Y. Shih, "Coverage analysis of cognitive femtocell networks," IEEE Wireless Communications Letters, vol. 3, no. 2, pp. 177-180, 2014.

[12] X. Ge, J. Martinez-Bauset, V. Gasares-Giner, B. Yang, J. Ye, and M. Chen, "Modeling and performance analysis of different access schemes in two-tier wireless networks," in Proceedings of the Global Communication Conference Exhibition and Industry Forum (Globecom '13), pp. 4402-4407, 2013.

[13] X. Ma, Y. Cao, Y. Liu, and K. S. Trivedi, "Modeling and performance analysis for soft handoff schemes in CDMA cellular systems," IEEE Transactions on Vehicular Technology, vol. 55, no. 2, pp. 670-680, 2006.

[14] J. Norris, Markov Chains, Cambridge University Press, Cambridge, UK, 1998. 


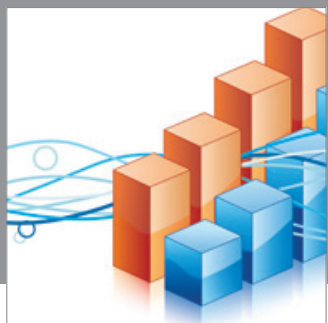

Advances in

Operations Research

mansans

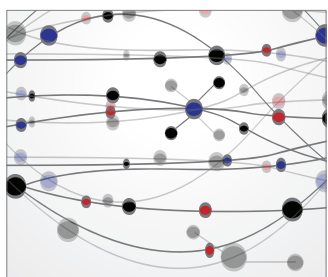

The Scientific World Journal
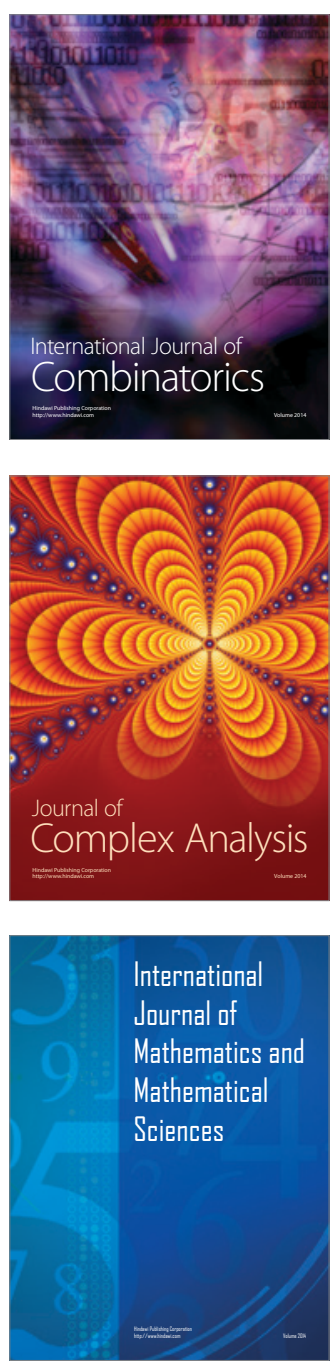
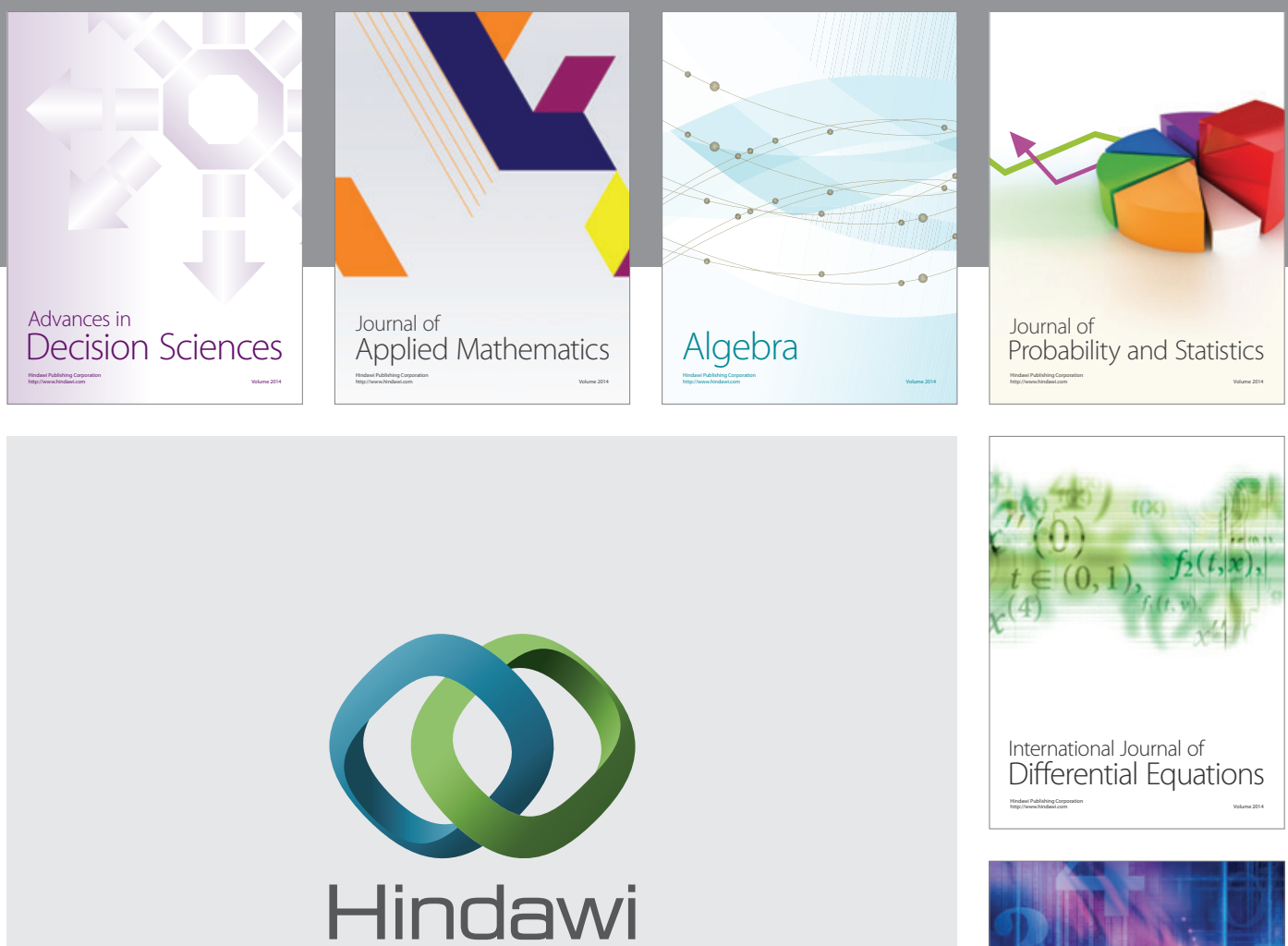

Submit your manuscripts at http://www.hindawi.com
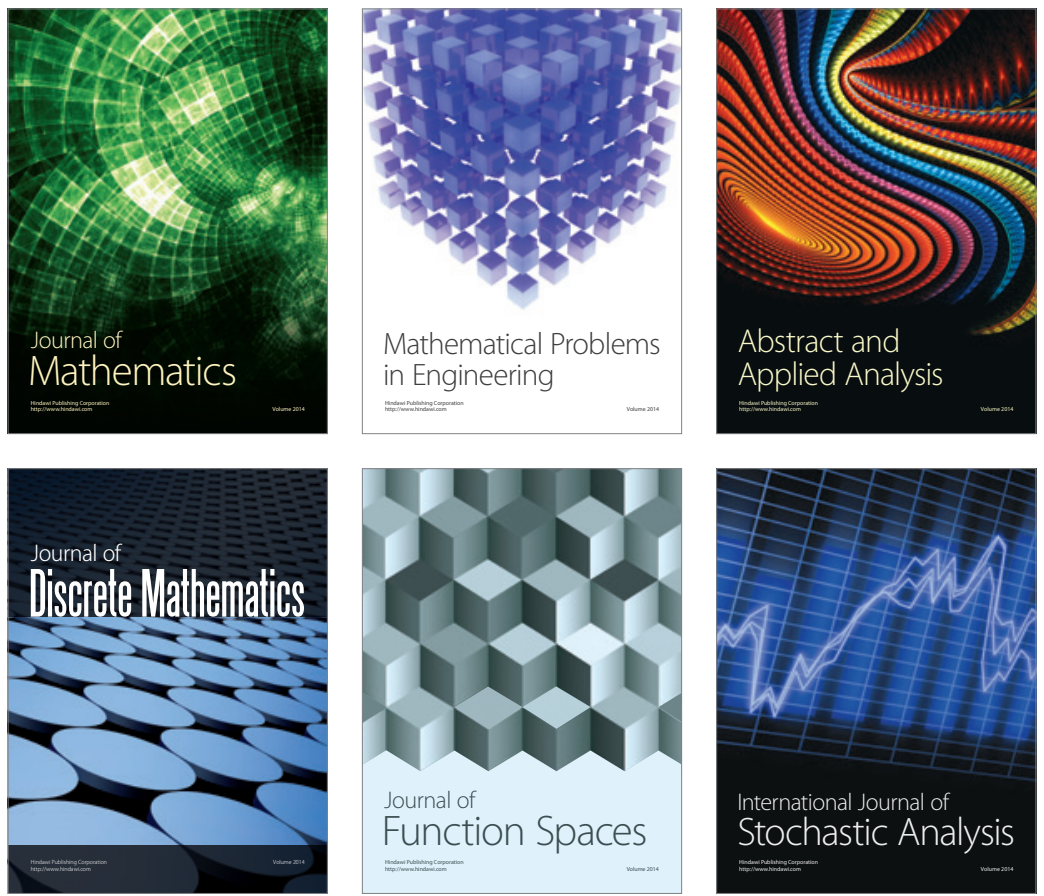

Journal of

Function Spaces

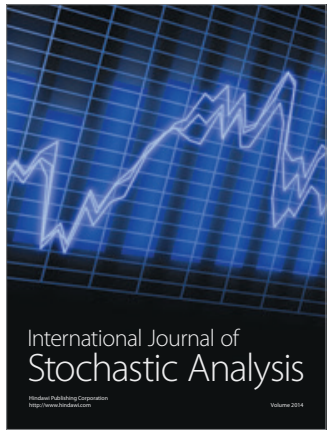

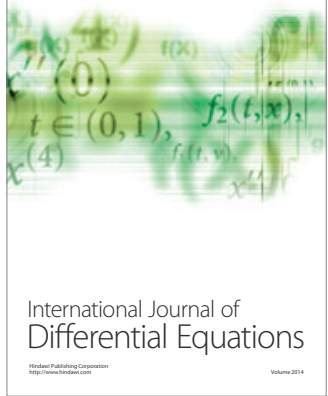
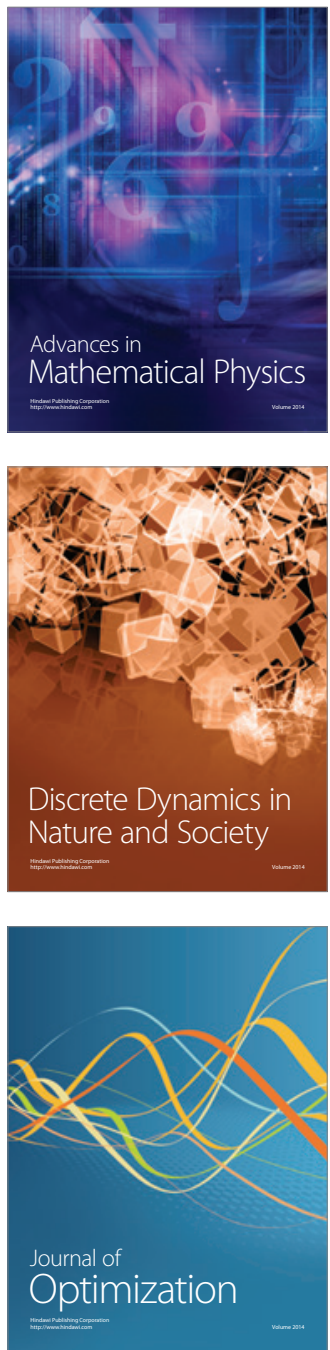